\title{
Strategic Health Economics Study Applied to Policymaking on the Prevention and Control of Chronic Non-Communicable Diseases
}

\author{
Gu Xuefei, Zhang Meili ${ }^{2}$ Liu Xiaoqing ${ }^{3}$, Li Tingting ${ }^{1}$, Huang Xiao ${ }^{4}$, Wang Chaoqun ${ }^{5}$, Xu Nan ${ }^{1}$, Xiang Guochun $^{1}$, \\ Liu Kejun ${ }^{1}$, Gao Runlin ${ }^{6}$
}

1. China National Health Development Research Center, Beijing 100191, China

2. School of Public Health and Management, Wenzhou Medical University, Wenzhou 325035, Zhejiang, China

3. School of Economics, Tianjin University of Finance and Economics, Tianjin 300222, China

4. School of Humanities and Management Sciences, Southwest Medical University, Luzhou 646000, Sichuan, China

5. School of Public Management, Central China Normal University, Wuhan 430079, China

6. Fuwai Hospital, Chinese Academy of Medical Sciences, Beijing 100037, China

\begin{abstract}
To improve public health by more effectively curbing the prevalence of chronic non-communicable diseases, the Chinese Academy of Engineering organized a major advisory project in 2015: Strategic Health Economics Study Applied to Policymaking on the Prevention and Control of Chronic Non-Communicable Diseases. This study demonstrated health economics to be an important decision-making tool that may be applied to the prevention and control of chronic non-communicable diseases while they remain in a primary stage. However, a lack of understanding of the importance of health economics applications and limitations in mastering and using health economics methods restricts the application of this tool in policymaking on the prevention and control of chronic noncommunicable diseases. We suggest multi-subject participation in health economics studies should be improved in the future, big data related to health economics accumulated and applied in policymaking on the prevention and control of chronic non-communicable diseases, and the strategic framework of health economics developed and applied to these policies as well.
\end{abstract}

Keywords: prevention and control of chronic non-communicable diseases; health economics; economic burden of disease; big data; strategic planning

\section{Introduction}

Worldwide, $68 \%$ of deaths are due to chronic disease and the corresponding proportion is as high as $86.6 \%$ in China. When chronic diseases become a major threat to residents' health, issues related to the resources that the government invested in the prevention and control of chronic diseases should be evaluated from the perspective of health economics, such as whether the resources meet the needs to chronic diseases prevention and control (CDPC), whether the allocation is efficient, and whether it improves fairness.

In 2015, the Chinese Academy of Engineering organized a major consulting project called "Strategic Health Economics Study Applied to Policymaking on the Prevention and Control of Chronic Non-Communicable Diseases." The research group selected five provinces/cites, namely, Shandong, Anhui, Sichuan, Chongqing, and Qinghai, and investigated the application of health economics in policymaking about chronic diseases

Received date: January 6, 2017; Revised date: February 25, 2017

Corresponding author: Gao Runlin, Fuwai Hospital, Chinese Academy of Medical Sciences, Professor; Chinese Academy of Engineering, Academician. Major research field is angiocardiopathy. E-mail: gaorunlin@citmd.com

Funding program: CAE Advisory Project "Strategic Health Economics Study Applied to Policymaking on the Prevention and Control of Chronic Non-Communicable Diseases" (2015-XZ-19)

Chinese version: Gu Xuefei et al. Strategic Health Economics Study Applied to Policymaking on the Prevention and Control of Chronic Non-Communicable Diseases. Strategic Study of CAE, https://doi.org/10.15302/J-SSCAE-2017.02.015 
prevention and control, trying to identify related problems and constraints, based on which we propose a strategy design to strengthen the application of health economics in policymaking.

\section{Current research on health economics applied to CDPC policymaking}

\subsection{Epidemic situation and economic burden of chronic diseases in China}

In 2015, there were about 4.3 million new cases of cancer and 2.8 million cancer patients died [1]. In the entire country, about 300 million people were suffering from high blood pressure, $11.6 \%$ of adults suffered from diabetes (as opposed to the $9 \%$ world average), $38.2 \%$ of Chinese suffered from sleep disorders, and 173 million from poor mental health [2]. The fifth national health services survey showed that the chronic diseases prevalence was $24.5 \%$ in adults aged 15 years and above, with $26.3 \%$ in cities and $22.7 \%$ in rural area, Morbidity was highest in eastern China, including cities and rural areas [3].

If there is no effective intervention from the government, chronic diseases will lead to large economic losses [4]. Taking cardiovascular disease for instance, in 2014, among hospital expenses, acute myocardial infarction costed 13.375 billion yuan, intracranial hemorrhage 20.707 billion yuan, and cerebral infarction 47.035 billion yuan, with an annual growth rate of $32.02 \%$, $18.9 \%$, and $24.96 \%$, respectively, since 2004 [5].

Due to the extremely high possibility and tremendous potential of causing serious economic losses, chronic diseases have been considered major risks to global economic development by the World Economic Forum in 2010 and 2011 consecutively. Therefore, the World Economic Forum suggested governments should formulate effective policies and national planning to cope with this risk [4].

\subsection{Current financing for chronic disease prevention and control}

The total health expenditure means the monetary resources invested by a country or region in the health system over one year. In 2012, for example, China's total expenditure for prevention services was 166.934 billion yuan, of which the 27.458 billion yuan for the immune programming accounted for $16.45 \%$ and the 7.093 billion yuan for CDPC accounted for $4.25 \%$ [6].

In $2012,75.59 \%$ of China's recurrent health expenditure was used for treatment, of which $44.66 \%$ for inpatient services, $30.93 \%$ for outpatient services, $6.74 \%$ for prevention service. In the cost for prevention services, unplanned immune costs accounted for $11.29 \%$, physical examination $11.35 \%$, and family planning $10.04 \%$. After deducting the latter three costs, less than $5 \%$ was used for prevention [6].

In 2012, Chinese residents' treatment expenses amounted to
2308.517 billion yuan, including 1575.62 billion for the treatment of chronic non-communicable diseases, accounting for $68.25 \%$. Diverse systemic diseases, such as those of the circulatory system; respiratory system; tumors; digestive tract; and disease of endocrine, nutritional, and metabolic nature, are the top five diseases consuming most medical resources and accounting for $56.83 \%$ of treatment costs [6].

\subsection{Application and problems of health economics in CDPC policymaking}

The policymaking on CDPC involves some important aspects, such as the identification of major diseases and their different intervention policies, allocation of resources, mechanisms of financial input and sharing responsibility, and evaluation of effects.

In the survey areas, we found health economics and related methods were rarely used to allocate resources in practice. In disease prevention control institutions at the levels of provinces, cities, and counties, personnel numbers in chronic diseases departments is limited by institutional establishment, accounting for less than $5 \%$ of the entire personnel, which does not reach the national requirement level of personnel standard configuration.

The survey found there were three problems in the application of health economics to community diagnosis. First, the intervention measures in all areas are similar or did not develop targeted intervention measures and models according to regional situations. Second, evaluation of results was not conducted after intervention. Community diagnosis is a process of circulation, the results of the last round of evaluation providing reference for the next round of community diagnosis, but several areas did not evaluate the intervention effect of the former. Parts of areas did so, but more likely focused on descriptions excluding cost analysis, namely without a complete health economics evaluation (HEE). Finally, effect indicators are often used to evaluate outcomes, but cannot be used for single-item evaluation or comparative analysis of different items. To overcome this problem, we should introduce the cost-benefit and cost-utility analyses, but they are not used in community diagnosis nowadays.

Chronic disease monitoring technologies and monitored contents are in accordance with the requirements of administrative management and have been proven effective and economic, which provides reference and assessment criteria to basic units Considering their own health resources, most basic units practice based on documents from higher authorities, while few improved their working processes or introduced HEE to chronic disease management and monitoring. All prevention institutions and health administrative agencies have realized the heavy economic burden of chronic diseases but did not use health economics to analyze it because of this economic burden.

Health economics evaluations for CDPC prioritize outcome 
evaluation. Policymakers need instant theory and data support when faced with abrupt problems, which cannot be coped with through health technology assessment (HTA) based on a research project that requires a long period to draft a policy. Therefore, finding the conjunction between HEE according to the principle that "soft fire makes sweet malt" and policymaking pursuing timeliness would be difficult point in applying health economics research to policymaking.

\section{Strategic health economics design applied to CDPC policymaking in China}

Based on the current status that the application of health economics mainly focuses on HTA at home and abroad, we propose the design of the application should be based on existing HTA, combined with the characteristics of CDPC practice, so that we can construct a systematic framework adapted to China's actual conditions.

\subsection{Basic orientation of health economics applied to CDPC policymaking}

First, CDPC policymaking should focus on "prevention." Research in China and abroad proved that effective intervention to controllable risk factors could prevent chronic diseases, which will not only promote the health of a nation's population but also bring higher economic benefits. In the long run, with a certain amount of funds, when the "prevention" achieves the goals of cost-effectiveness and cost-benefit, the funds used to "control" it will relatively decrease, reaching overall effectiveness and benefitting prevention and control. Therefore, the application of health economics in CDPC should "prevent predominantly, control efficiently."

In the future, the application of health economics to CDPC should concentrate mainly on two aspects (Fig. 1): The first is how to effectively allocate and utilize limited health resources to get maximum satisfaction from individuals' needs and demand for CDPC and improve economic and social benefits. That is, we should pay attention to "input" issues, such as the amount and allocation structure of resources used for "prevention." The second aspect is evaluating the effectiveness of policies on prevention and control and the results of resource allocation. In other words, we should pay attention to "output" issues, such as evaluation after intervention and warning systems. The two aspects are closely linked, mutually guide and provide feedback, as well as mutually reinforce each other.

\subsection{Strategic framework of health economics applied to CDPC policymaking}

\subsubsection{Vision}

Developing the value of health economics applied to the CDPC provides a solid foundation for health economics to be applied to China's overall public health service system and promotes the achievement of scientification and innovation of the public health service system in China.

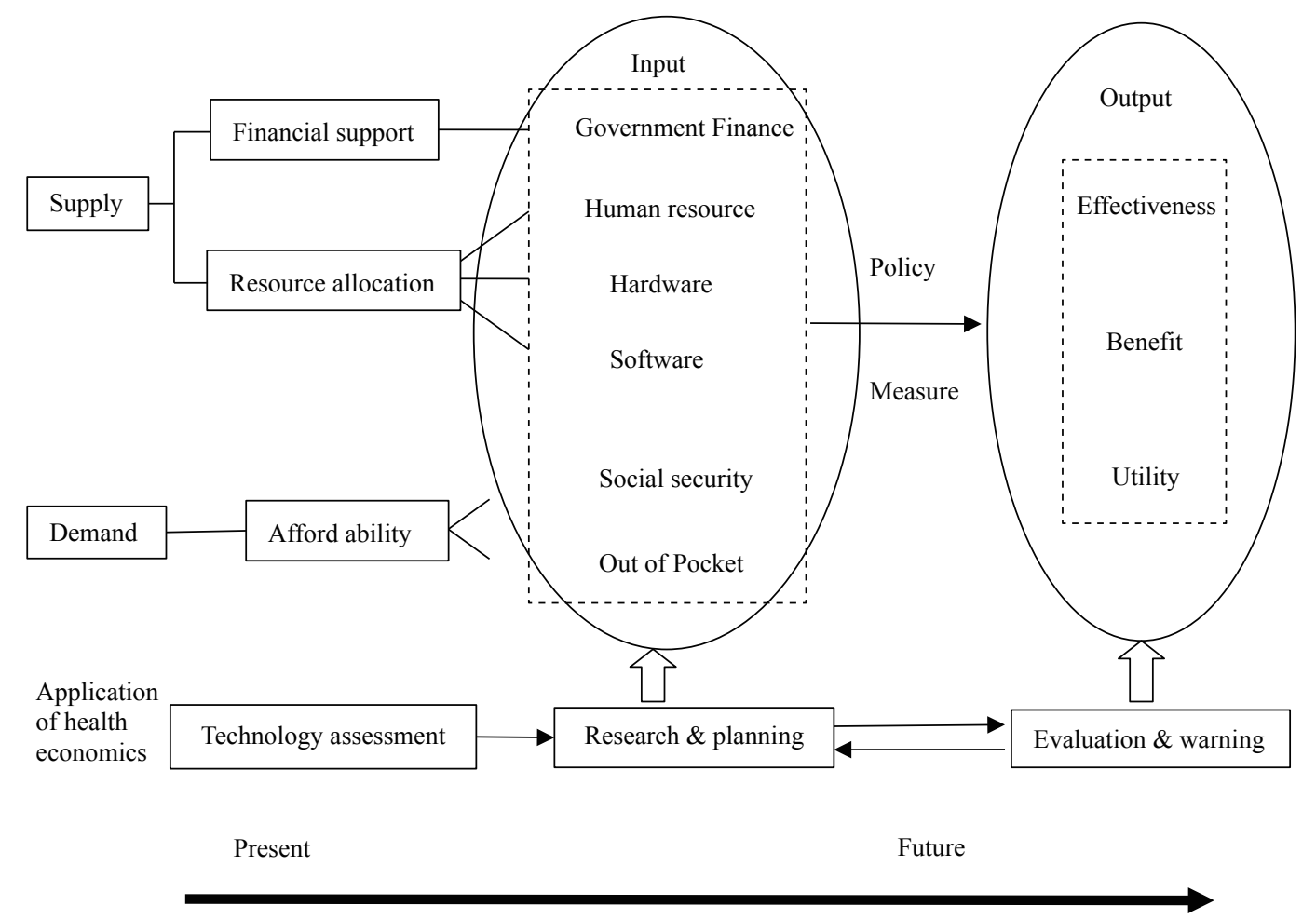

Fig. 1. Concerns of health economics applied to CDPC policymaking. 


\subsubsection{Strategic orientation}

Health economics should be implemented in the entire process of policymaking and CDPC implementation. We should thus conduct theoretical analysis, field research, and policy planning before decision making to forecast decision effect and problems at the beginning of execution, track the cost-effectiveness and cost-benefit in the execution process and evaluate its effect, estimate benefits and total utility after a period of time to provide references for policy amendments or reform.

\subsubsection{Strategic goals}

Combing health economics with the latest economic and science developments, with the use of big data and "Internet + Health," we develop a standard evaluation system of health economics applicable to CDPC policymaking in China.

\subsubsection{Strategic framework diagram}

The strategic framework diagram of health economics applied to CDPC policymaking is shown in Fig. 2.

\subsection{Possible impetus and opportunities for strategy promotion}

\subsubsection{Large potential demand for CDPC as directive power}

According to the analysis of China's chronic disease morbidity and potential threats, we find that a pressing issue of CDPC is how to achieve high efficiency and benefits. However, in the situation of limited financial power of the both central and local governments, the issues of ensuring resources are fairly distributed, policies effectively implemented, and good social benefits created cannot be resolved only by technology assessment and optimization. Therefore, we should encourage the government and public health department to introduce knowledge and methods from health economics, evaluate special disease intervention, and achieve the rationalization of resource amounts and allocation structure.

3.3.2 In-step development of health economics application and CDPC as a key opportunity

Presently, China focuses on CDPC and, with the new health reform, China pays increasing attention to this subject, but systematic practice is still developing. Health economics has been applied to chronic diseases but mainly on technology assessment, lacking evaluations in terms of cost efficiency and economic benefits. As such, there is room to improve the application of health economics in CDPC. Recently, the concerns of the government and public have been gaining ground, which provides a key opportunity for the in-step development of CDPC and health economics applications and decreases the potential obstacles to the connection between systems and methods. Urgency of this systematic framework puts forward the theoretical construction and application of health economics.

3.3.3 Health technology assessment as the foundation for the application of health economics to CDPC

The main applicability of health economics is embodied in health technology assessment (HTA), which is significant for CDPC. On one hand, HTA could improve quality and, on the other hand, it upgrades the scientific degree of health policy research. More importantly, using health economics methods to

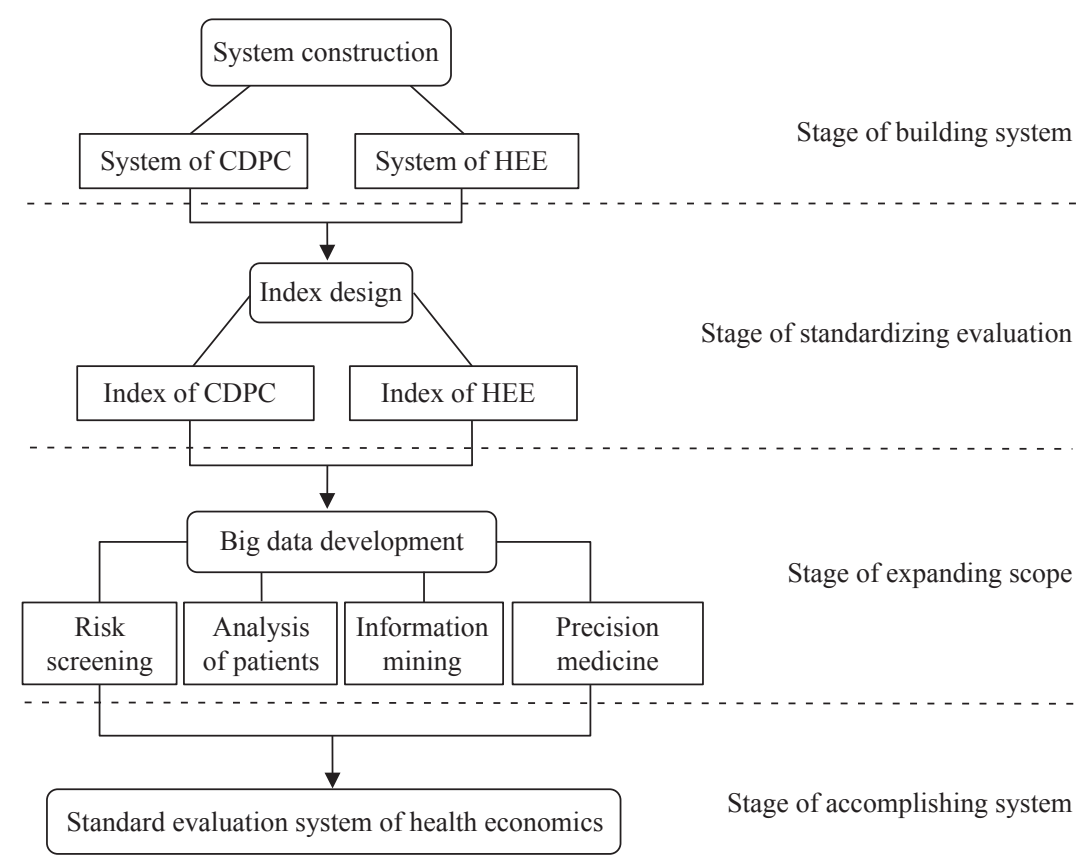

Fig. 2. Strategic framework diagram of health economics applied to CDPC policymaking. 
evaluate the effectiveness of health services and technology has become main stream in current HTA, and reached consensus among policymakers and researchers. Therefore, we can consider HTA as a foundation to develop the research system of health economics applied to CDPC.

\subsubsection{China's relatively complete CDPC network}

In China, there are four stakeholders in the CDPC system: research institutions, policymaking agencies, policy execution agencies, and the public or patients. The former three involves policy planning, evaluation, and feedback, which is the main body of the network of CDPC (Fig. 3), with related departments including Ministry of Science and Technology, Ministry of Finance, National Development and Reform Commission, Ministry of Human Resources and Social Security, and their subordinate agencies, all levels of government, publicity departments, and organization departments. These departments have a great ability for mobilization and research and would be a source of strength for health economics application.

3.3.5 Big data and "Internet + Health" bring an important opportunity period

The age of big data and "Internet + Health" will not only bring tremendous support for data sources, but also stimulate more institutions and talents to develop evaluation indicators and a standard system of health economics. In this context, information mining, risk screening, precision medicine will accomplish great progress.

\subsection{Possible resistance and dilemmas}

3.4.1 Knowledge and practice of health economics are not mastered by grassroots departments

In China, most healthcare fields pay attention to the process of prevention, while ignoring policy concepts, standards and aims, and health economic research on outcome evaluation, feedback and future development because policymakers and executors are not familiar with health economics. Lack of knowledge and methods directly hinder its application, which induces current prevention and control to retaining non-permanent models, with vague aims and effects.

3.4.2 Cooperation mechanisms between and within departments are insufficient

Executive agencies tend to mechanically follow superior commands, showing lack of initiative and autonomy. Further, there is limited motivation between parallel departments to connect each other. Health providers and centers for disease control (CDCs) cannot communicate effectively, and the former focuses on treatment without paying attention to chronic disease prevention, while CDCs do not set incentive mechanisms for health providers. All these situations do not improve the application of health economics.

3.4.3 Data is not accessible due to insufficient information construction

First, there are serious issues related to the phenomena of health care information islands, which block data inter-flow.
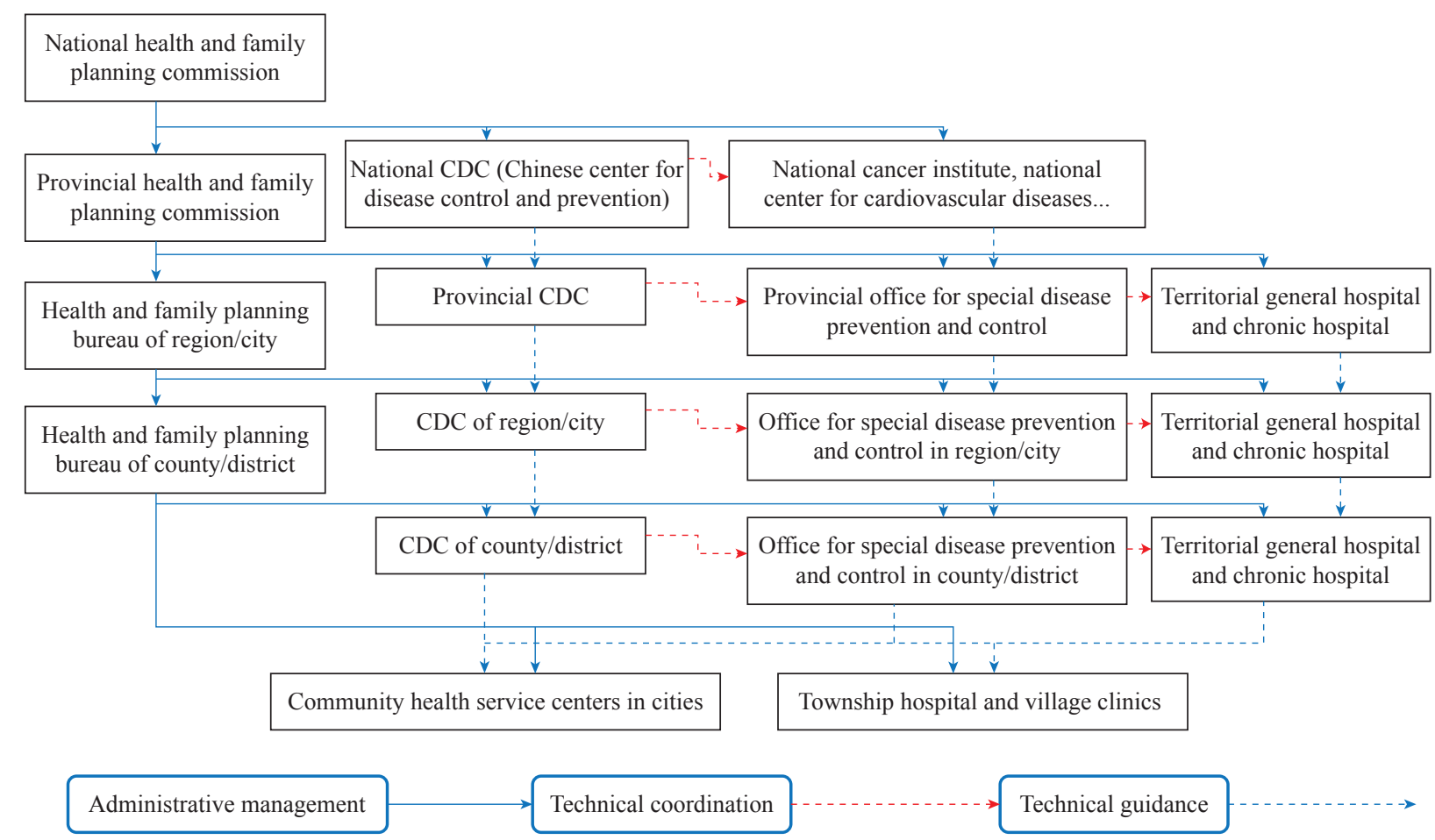

Fig. 3. China's work network diagram for CDPC. 
Second, the lack of specific statistical data on expenses leads to failure in systematizing cost-effectiveness analysis. Further, limited data resources cannot support the development of evaluation indicators and standards, which hinders the development of the HEE system applied to CDPC.

\subsection{Essential points for application of health economics to policymaking about CDPC}

\subsubsection{Gather research powers from multiple divisions}

Based on the current network, we should encourage and lead more and more institutions and individuals to do research and practice of health economics (Fig. 4). It is very important to set up a national HEE center.

\subsubsection{Improving the systematization and execution of policies}

Health economics will not emphasize its application value until a system is set up for CDPC. Accordingly, how to strengthen the execution power of CDPC policies becomes an urgent matter. We should thus reinforce CDPC, integrate the influence of all departments and associations, and enhance public engagement.

\subsubsection{Creating a national database for CDPC}

We should progressively integrate current CDPC databases to achieve a national uniform database.
Meanwhile, we should take advantage of big data and "Internet + Health," identify and gather important data sources (Table 1), enlarge the application fields of health economics, and upgrade its value.

\section{Conclusions}

Health economics could supply important technology support for optimizing health resources, choosing diseases with high priority, and evaluating current CDPC policies, as it has significance in dealing with chronic disease prevalence and improving public health. Chronic diseases are a significant threat to population health and induce heavy economic burden, so the prevalence of chronic disease is an important public health problem, while how to allocate limited health resources to CDPC is a difficult problem for governments. Therefore, applying health economics to CDPC will be a significant attempt.

\section{References}

[1] Chen W Q, Zheng R S, Baade P D, et al. Cancer statistics in China, 2015 [J]. CA: A Cancer Journal for Clinicians, 2016, 66(2): $115-132$.

[2] National Center for Cardiovascular Diseases, China. Report on

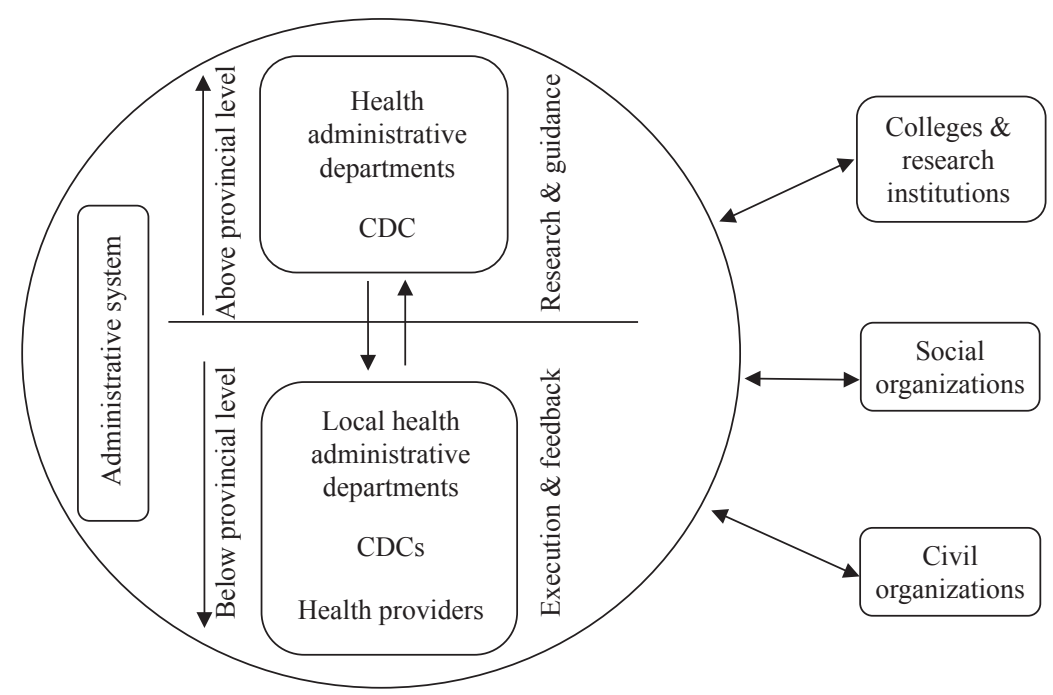

Fig. 4. Multiple participation system for application of health economics to CDPC.

Table 1. Critical data sources for mining big data on health economics [7].

\begin{tabular}{lc}
\hline Critical data sources & Examples \\
\hline Government regulators & Policy and data \\
Health providers & Electronic medical record, clinical test results, clinical evaluation records, etc. \\
Health insurance agencies & Claims data, general information of the insured, etc. \\
Patients & Social network, behavioral data, patients' effect data, etc. \\
Pharmaceutical factories & Marketing data, health care data, quantity of R\&D, etc. \\
\hline
\end{tabular}


cardiovascular diseases in China (2014) [M]. Beijing: Encyclopaedia of China Publishing House, 2015. Chinese.

[3] China National Health Statistical Information Center. The fifth analysis report of national health services survey in China, 2013 [M]. Beijing: Peking Union Medical College Press, 2015. Chinese.

[4] World Bank. Toward a healthy and harmonious life in China: Stemming the rising tide of non-communicable diseases [EB/ OL]. (2011-07-26) [2016-12-28]. Chinese. http://documents. worldbank.org/curated/en/621841468023051158/pdf/634260REVISED00UBLIC00ncd0report0cn.pdf.
[5] Wang L D. Prevention and control of chronic non-communicable diseases and health China [EB/OL]. (2016-10-14) [2016-11-15]. http://www.ncd.org.cn/Article/index/id/6459. Chinese.

[6] China National Health Development Research Center. Green book on China health development: Research on healthcare reforms [M]. Beijing: People's Medical Publishing House, 2015. Chinese.

[7] Xuan J W. The establishment of the big medical database and its application for the evidence-based medicine [C]. Shanghai: The 10th China Health Technology Assessment Forum, 2016. Chinese. 